\title{
Measurement of operative femoral anteversion during cementless total hip arthroplasty and influencing factors for using neck-adjustable femoral stem
}

Jingyang Sun ${ }^{1,2+}$, Bohan Zhang ${ }^{1,2+}$, Lei Geng ${ }^{2}$, Qingyuan Zheng ${ }^{1,2}$, Juncheng $\mathrm{Li}^{1,2}$, Wenzhe CaO ${ }^{2}$, Ming Ni $\mathrm{Ni}^{1,2^{*}}$ and Guoqiang Zhang ${ }^{1,2^{*}}$

\begin{abstract}
Background: Placement of femoral stem in excessive anteversion or retroversion can cause reduced range of motion, prosthetic impingement, and dislocation. The aim of this study was to assess the operative femoral anteversion in patients treated with total hip arthroplasty (THA) and analyze the need of adjusting stem anteversion.

Methods: We retrospectively included 101 patients (126 hips) who underwent cementless THA with a manual goniometer to determine the femoral anteversion between October 2017 and December 2018. The operative femoral anteversion we measured was recorded during THA. We further divided those hips into three subgroups based on the range of operative femoral anteversion: group $1\left(<10^{\circ}\right)$, group $2\left(10-30^{\circ}\right)$, and group $3\left(>30^{\circ}\right)$ and compared the differences of their demographic data. Univariate and multivariate logistic regression were used to identify the influencing factors for the need of neck-adjustable femoral stem. The clinical and radiographic outcomes were also assessed. Perioperative complications were recorded.

Results: After THA, the Harris hip scores improved from $52.87 \pm 15.30$ preoperatively to $90.04 \pm 3.31$ at the last follow-up ( $p<0.001$ ). No implant loosening, stem subsidence, and radiolucent lines were observed on radiographs. No severe complications occurred and no components needed revision at the latest follow-up. The mean operative femoral anteversion was $14.21^{\circ} \pm 11.80^{\circ}$ (range, -9 to $60^{\circ}$ ). Patients with femoral anteversion more than $30^{\circ}$ were about 10 years younger than others. Femoral anteversion $>30^{\circ}$ was more common in patients with developmental dysplasia of the hip (DDH). There were totally 14 hips treated with the neck-adjustable femoral stem. From the univariate analysis, we can observe that female sex, diagnosis of DDH (compared with osteonecrosis), and higher operative femoral anteversion and its value $>30^{\circ}$ (compared with $<10^{\circ}$ ) are associated with higher rates of using the neck-adjustable femoral stem. However, all these factors were no longer considered as independent influencing factors when mixed with other factors.
\end{abstract}

\footnotetext{
*Correspondence: niming301@163.com; gqz301@126.com

${ }^{\dagger}$ Jingyang Sun and Bohan Zhang contributed equally to this work and are considered co-first authors.

${ }^{1}$ Medical School of Chinese PLA, Beijing 100853, China

Full list of author information is available at the end of the article
} changes were made. The images or other third party material in this article are included in the article's Creative Commons licence, unless indicated otherwise in a credit line to the material. If material is not included in the article's Creative Commons licence and your intended use is not permitted by statutory regulation or exceeds the permitted use, you will need to obtain permission directly from the copyright holder. To view a copy of this licence, visit http://creativecommons.org/licenses/by/4.0/. The Creative Commons Public Domain Dedication waiver (http://creativecommons.org/publicdomain/zero/1.0/) applies to the data made available in this article, unless otherwise stated in a credit line to the data. 
Conclusions: This study highlighted the significance of operative femoral anteversion. Identification of abnormal femoral anteversion could assist in adjusting stem anteversion and reduce the risk of dislocation after THA.

Keywords: Femoral anteversion, Total hip arthroplasty, Operative, Measurement

\section{Background}

Proper positioning of both acetabular and femoral components can lower the rate of impingement, dislocation, and accelerated wear in patients with total hip arthroplasty (THA) [1-3]. Many studies focused on the orientation of the acetabular component in THA, but little has been conducted regarding the position of the femoral component [3-5]. Even though the stem version is not as important as the cup version after THA, placement of femoral stem in excessive anteversion or retroversion can cause a clinically relevant reduction in range of motion and increase in the incidence of dislocation [6]. Since more postoperative dislocation occurred within Lewinnek's safe zone, the concept of combined anteversion gained its popularity $[5,7,8]$. No matter femur first or cup first technique, it is necessary to know the native anteversion of the femur before implanting the femoral stem.

Several methods have been introduced to determine the femoral anteversion. The most commonly used clinical method to assess femoral anteversion is Craig's test, which is based on differences between medial and lateral rotation on the extended hip [9]. The means of radiographic evaluation contains $\mathrm{X}$-ray under special position and CT and MRI scan [10-12]. The latter two which involve the profile of the distal femur are thought to be more accurate and comprehensive. However, the preoperative evaluation pays more attention to the femoral neck anteversion, which is not equal to the torsion of the intramedullary canal $[13,14]$. Therefore, even knowledge of the neck anteversion based on CT scan, it would be better to measure the version on the cutting surface of the femoral neck during operation.

The reference axis of the distal femur cannot be directly visualized during THA, so surgeons usually orient the lower leg perpendicular to the floor as a surrogate for the posterior condylar axis in order to measure the femoral anteversion [15, 16]. Unlike the definitions of anatomical, radiographic, and operative acetabular anteversion by Murray et al., there has been no specific classification of femoral anteversion [17]. When measuring the femoral anteversion with this method, we prefer to call it "operative anteversion" to distinguish from femoral neck anteversion. However, several studies demonstrated that visual estimation of the femoral anteversion had poor precision even for experienced surgeons [18, 19]. Thereafter, manual goniometers of different kinds with the same measuring principle have been reported, with an acceptable absolute error $[15,16,20]$. But these goniometers also relied on the premise that the lower leg axis was vertical to the reference axis of the distal femur, and recent studies showed that knee osteoarthritis could increase the error in estimating femoral anteversion [16, 21]. It must be acknowledged that navigation can assess femoral anteversion with high accuracy [7, 22]. However, the navigation is not available to most orthopedic surgeons and has the disadvantages of prolonged operative time and higher costs.

We designed a goniometer to measure the operative anteversion of the femur on the cutting surface during THA. We used it to identify the abnormal femoral anteversion and further to guide the implantation of the femoral stem. Therefore, the aim of this study was to assess the operative femoral anteversion in patients treated with THA and analyze the need of adjusting stem anteversion.

\section{Patients and methods Patients}

We retrospectively reviewed 101 patients (126 hips, 76 hips of unilateral cases and 50 hips of bilateral cases) who underwent cementless THA with a goniometer to determine the femoral anteversion between October 2017 and December 2018. Inclusion criteria were primary THA, severely symptomatic hip, and lowered life quality. Patients with severe angulation deformity of the femur, active infection, or advanced knee osteoarthritis were excluded. Within this cohort, the diagnosis was osteoarthritis secondary to developmental dysplasia of the hip (DDH) in 39, osteonecrosis of the femoral head in 49, ankylosing spondylitis in 19, rheumatoid arthritis in 6, slipped epiphysis of the femoral head in 6 , posttraumatic arthritis in 1 , sequelae of hip pyogenic arthritis during childhood in 1 , and displaced femoral neck fracture in 5. According to Crowe classification for DDH, 19 hips were type I, 11 hips were type II, 5 hips were type III, and 4 hips were type IV. There were 66 males and 60 females. The mean age was $50 \pm 14$ years (range, 2385 years). The mean body mass index (BMI) was $24.35 \pm 3.78 \mathrm{~kg} / \mathrm{m}^{2}$ (range, $15.55-35.49 \mathrm{~kg} / \mathrm{m}^{2}$ ). There were 71 left hips and 55 right hips. Informed consent was obtained by all patients. The study was approved by the institutional review board and conducted according to the Declaration of Helsinki principles. 


\section{Surgical procedure}

All surgeries were performed by two senior arthroplasty surgeons under general anesthesia through a posterolateral approach. Standardized preoperative planning of the prosthesis size and position was performed based on the plain radiographs. We adopted the "cup first" technique in all hips. Aiming for secure press-fit fixation, the acetabular cup was implanted with as much host bone coverage as possible. The target orientation of the cup was $40^{\circ} \pm 5^{\circ}$ inclination and $25^{\circ} \pm 5^{\circ}$ anteversion.

When preparing the femoral side, the surgeon measured the anteversion of the cutting surface with a manual goniometer after the femoral neck osteotomy. The goniometer consisted of three parts: one end of the handle was placed along the long axis of the cutting surface; the other end was a calibrated scale providing information for the orientation; and a laser device was attached to the pointer, which irradiated a ray of light to position the lower leg axis (Fig. 1). The scrub technician flexed the knee and hip and internally rotated the hip until the lower leg was vertical to the operative table. In this position, the angle between the axis of the lower leg and the

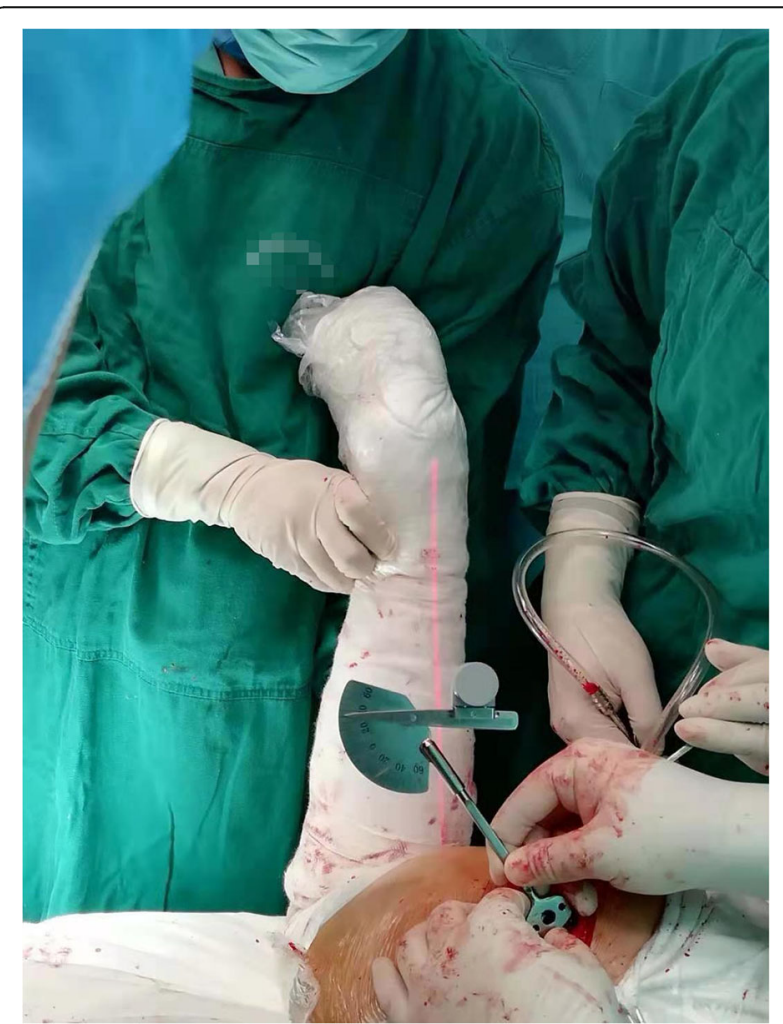

Fig. 1 Photograph showing intraoperative measurement of the femoral anteversion using a goniometer. The goniometer consisted of 3 parts: one end of the handle was placed along the long axis of the cutting surface; the other end was a calibrated scale providing information for the orientation; and a laser device was attached to the pointer, which irradiated a ray of light to position the lower leg axis long axis of the cutting surface (midcortical line) was measured using the goniometer and its coangle (subtracted by $90^{\circ}$ ) was pointed on the scale indicating the femoral anteversion, which we called operative anteversion. When the femoral anteversion indicated a superior position in reference to the table, it was defined as a positive value.

Based on the target combined anteversion of $30-50^{\circ}$ and approximate operative anteversion of cup, we assessed the difference between the measured femoral anteversion and the anticipated stem anteversion. In our opinion, a difference less than $10^{\circ}$ can be addressed by orienting the broach version with a box chisel or rasp. Difference more than $10^{\circ}$ increased the possibility of using a femoral stem with an optional neck version or even a modular stem. After inserting the femoral trial, the combined anteversion was measured by a coplanar test, and the soft tissue tension was also assessed. Finally, we would ensure that the stem and cup were fine-tuned with an impingement-free range of motion. The prostheses used in this cohort were shown in Table 1.

Patients were allowed to walk with crutches on the first postoperative day. All patients received postoperative intravenous antibiotic prophylaxis with thirdgeneration cephalosporins. Low molecular weight heparin was also administered as antithrombotic prophylaxis.

\section{Clinical and radiographic assessment}

Patients were asked for a follow-up visit in regular intervals at 3 months, 6 months, and yearly after surgery. Clinical and radiographic assessments were performed at each visit. Clinical outcome was evaluated using the Harris hip score. Standardized digital, calibrated anteroposterior and lateral hip radiographs were acquired. The presence of implant loosening, stem subsidence, and radiolucent lines was detected by two reviewers. All perioperative complications were recorded including dislocation, implant loosening, and infection. Failure was defined as revision for any reason.

\section{Statistical analysis}

Categorical variables were presented as frequencies and continuous variables as means and standard deviation. Paired t-test was used to evaluate differences between pre- and postoperative quantitative data. Categorical variables were analyzed using the chi-square test or Fisher's exact test. One-way ANOVA was carried out to explore the differences between the variables in the subgroups. Binary logistic regression was used to identify the influencing factors for using the neck-adjustable femoral stem. Odds ratios (ORs) and 95\% confidence intervals (CIs) were calculated for these results. All statistical analyses were performed using SPSS version 26.0 (IBM Inc., 
Table 1 Acetabular and femoral prostheses

\begin{tabular}{lll}
\hline Prostheses & Cases & Manufactures \\
\hline Acetabular cup & & Waldemar Link, Hamburg, Germany \\
Betacup & 60 & Waldemar Link, Hamburg, Germany \\
Combicup & 20 & DePuy Synthes, Warsaw, IN, USA \\
Pinnacle & 12 & Stryker, Mahwah, NJ \\
Trident & 21 & Stryker, Mahwah, NJ \\
Tritanium & 12 & LDK, Beijing, China \\
CDH cup & 1 & Waldemar Link, Hamburg, Germany \\
Femoral stem & & DePuy Synthes, Warsaw, IN, USA \\
LCU & 69 & DePuy Synthes, Warsaw, IN, USA \\
Corail & 4 & Stryker, Mahwah, NJ \\
S-ROM & 7 & LDK, Beijing, China \\
Accolade II & 39 & 7
\end{tabular}

Armonk, NY). $P$ values $<0.05$ were considered statistically significant.

\section{Results}

The mean duration of follow-up was $30.8 \pm 4.2$ months (range, 24-38 months). After THA, the Harris hip scores improved from $52.87 \pm 15.30$ preoperatively to $90.04 \pm$ 3.31 at the last follow-up $(p<0.001)$. No implant loosening, stem subsidence, and radiolucent lines were observed on radiographs. No severe complications occurred and no components needed revision at the latest follow-up.

The mean operative femoral anteversion was $14.21^{\circ} \pm$ $11.80^{\circ}$ (range, -9 to $60^{\circ}$ ). A graph depicting the distribution of operative femoral anteversion is shown in Fig. 2. We further divided those hips into three subgroups based on the range of operative femoral anteversion: group $1\left(<10^{\circ}, 42 \mathrm{hips}\right)$, group $2\left(10-30^{\circ}, 74 \mathrm{hips}\right)$, and group $3\left(>30^{\circ}, 10 \mathrm{hips}\right)$. The differences of the demographic data in these three subgroups were compared

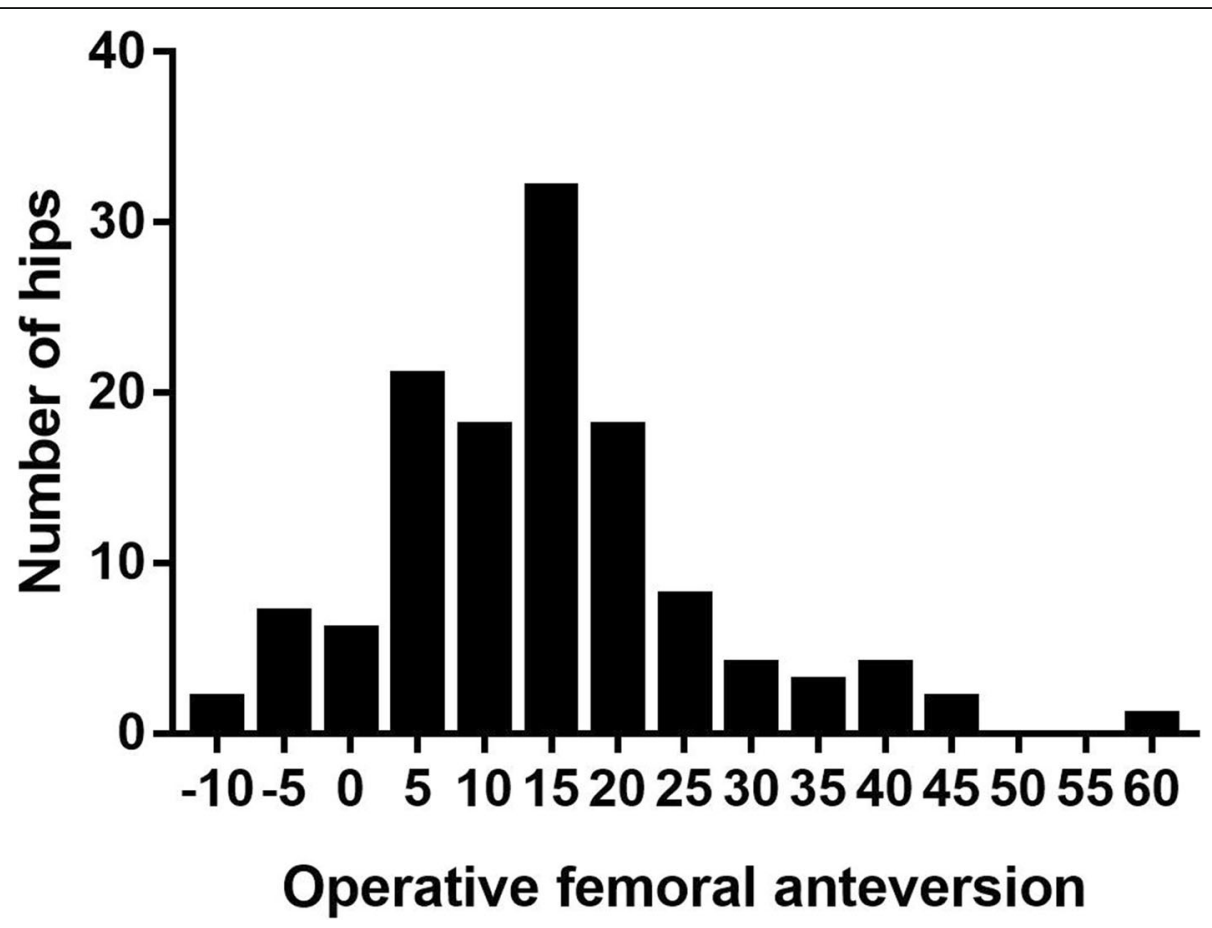

Fig. 2 Graph of the distribution of operative femoral anteversion measured with a manual goniometer during total hip arthroplasty 
and results are shown in Table 2. It can be seen that patients with femoral anteversion more than $30^{\circ}$ were about 10 years younger than others. With regard to different diagnoses, femoral anteversion $>30^{\circ}$ was more common in DDH patients. Half hips in group 3 were treated with the neck-adjustable femoral stem, which was obviously greater than the other two groups.

There were totally 14 hips treated with the neckadjustable femoral stem (including 7 S-ROM and 7 $\mathrm{CDH}$ stem with optional neck version). The results of analyzing the influencing factors for the need of neckadjustable femoral stem are shown in Table 3. From the univariate analysis, we can observe that female sex, diagnosis of DDH (compared with osteonecrosis), and higher operative femoral anteversion and its value $>30^{\circ}$ (compared with $<10^{\circ}$ ) are associated with higher rates of using the neck-adjustable femoral stem. However, in the multivariate analysis, all these factors were no longer considered as independent influencing factors when mixed with other factors.

\section{Discussion}

Correct component placement has been considered a prerequisite for successful THA, as implant malposition directly influences postoperative stability, wear, and aseptic loosening [1-3]. Even though the stem version is not as important as the cup version after THA, it can also influence the range of motion, bone loading, and gait $[6,23,24]$. Early identification of the abnormal femoral version can assist in obtaining optimal stem anteversion in THA. In this study, we used a manual goniometer to determine the operative femoral anteversion and further guide the implantation of the femoral stem. All patients had an evident improvement in clinical scores and no severe complications occurred. Though our goniometer is not as accurate as computer navigation, it is easily put to practical use and less invasive. Above all, knowledge of the operative femoral anteversion can remind us of the need to adjust stem anteversion, further lowering the rate of prosthetic impingement.

Our study has several limitations. First, we did not validate the accuracy of our goniometer with a postoperative CT scan, which was not routinely examined after THA. Moreover, the discrepancy between intraoperative estimation and measurement on CT did not actually represent the precision due to the potential rotational adjustment of the femoral stem $[25,26]$. Second, we did not concern about the actual posterior femoral condylar axis, but used the lower leg to approximate it. With the assumption that the lower leg is vertical to the posterior condylar axis, the orientation of its surface was not taken into consideration [15]. However, patients with advanced knee osteoarthritis were not included, which was the influencing factor for erroneous estimation. Third, no specific tools were available to measure the operative anteversion of the acetabular cup. We believe that it would not have much influence because of the error tolerance of our target combined anteversion [27, 28]. Fourth, although patients with various diagnoses were enrolled, the sample size of each might not be big enough.

The angle we called operative femoral anteversion is actually the torsional version on the cutting surface. We

Table 2 Characteristics of hips with different ranges of operative femoral anteversion

\begin{tabular}{|c|c|c|c|c|}
\hline & Group $1\left(<10^{\circ}, 42\right.$ hips $)$ & Group $2\left(10-30^{\circ}, 74\right.$ hips $)$ & Group $3\left(>30^{\circ}, 10\right.$ hips $)$ & $P$-value \\
\hline Age (years) & $53 \pm 12$ & $49 \pm 15$ & $40 \pm 11$ & $0.022^{\dagger, \pm}$ \\
\hline Height (m) & $1.66 \pm 0.09$ & $1.66 \pm 0.08$ & $1.63 \pm 0.09$ & 0.529 \\
\hline Weight (kg) & $66.8 \pm 12.7$ & $67.9 \pm 14.3$ & $64.4 \pm 8.5$ & 0.714 \\
\hline $\mathrm{BMI}\left(\mathrm{kg} / \mathrm{m}^{2}\right)$ & $24.09 \pm 3.49$ & $24.50 \pm 4.08$ & $24.26 \pm 2.80$ & 0.857 \\
\hline Female/male sex, $n$ & $16 / 26$ & $37 / 37$ & $7 / 3$ & 0.157 \\
\hline Diagnosis, n (\%) & & & & $0.032^{\dagger, \varepsilon}$ \\
\hline $\mathrm{DDH}$ & $10(23.8)$ & $22(29.7)$ & $7(70)$ & \\
\hline Osteonecrosis & $15(35.7)$ & $34(45.9)$ & $0(0)$ & \\
\hline Ankylosing spondylitis & $9(21.4)$ & $10(13.5)$ & $0(0)$ & \\
\hline Rheumatoid arthritis & $2(4.8)$ & $3(4.1)$ & $1(10)$ & \\
\hline Slipped epiphysis of the femoral head & $1(2.4)$ & $3(4.1)$ & $2(20)$ & \\
\hline Posttraumatic arthritis & $1(2.4)$ & $0(0)$ & $0(0)$ & \\
\hline Sequelae of hip pyogenic arthritis & $1(2.4)$ & $0(0)$ & $0(0)$ & \\
\hline Displaced femoral neck fracture & $3(7.1)$ & $2(2.7)$ & $0(0)$ & \\
\hline Use of the neck-adjustable femoral stem & $2(4.8)$ & $7(9.5)$ & $5(50)$ & $<0.001^{\dagger, £}$ \\
\hline
\end{tabular}

$B M I$ body mass index, $D D H$ developmental dysplasia of the hip

${ }^{*} p<0.05$ group 1 vs group $2 ;{ }^{\dagger} p<0.05$ group 1 vs group $3 ;{ }^{f} p<0.01$ group 2 vs group 3 
Table 3 Univariate analysis of the characteristics of hips treated with conventional and neck-adjustable femoral stem

\begin{tabular}{|c|c|c|c|c|}
\hline & Conventional stem (112 hips) & Neck-adjustable stem (14 hips) & OR $(95 \% \mathrm{Cl})$ & $P$-value \\
\hline Age (years) & $50 \pm 13$ & $48 \pm 18$ & $0.991(0.951-1.031)$ & 0.644 \\
\hline Height (m) & $1.66 \pm 0.08$ & $1.63 \pm 0.07$ & $0.963(0.897-1.033)$ & 0.292 \\
\hline Weight (kg) & $67.7 \pm 13.4$ & $63.9 \pm 12.8$ & $0.976(0.931-1.024)$ & 0.322 \\
\hline $\mathrm{BMI}\left(\mathrm{kg} / \mathrm{m}^{2}\right)$ & $24.42 \pm 3.76$ & $23.78 \pm 4.06$ & $0.955(0.819-1.113)$ & 0.552 \\
\hline Female/male sex, $\mathrm{n}$ & $48 / 64$ & $12 / 2$ & $8.000(1.710-37.430)$ & 0.008 \\
\hline Diagnosis, n (\%) & & & & 0.026 \\
\hline $\mathrm{DDH}$ & $30(26.8)$ & $9(64.3)$ & Reference & \\
\hline Osteonecrosis & $48(42.9)$ & $1(7.1)$ & $0.069(0.008-0.576)$ & 0.013 \\
\hline Ankylosing spondylitis & $19(17.0)$ & $0(0)$ & 0 & 0.998 \\
\hline Rheumatoid arthritis & $5(4.5)$ & $1(7.1)$ & $0.667(0.069-6.470)$ & 0.727 \\
\hline Slipped epiphysis of the femoral head & $4(3.6)$ & $2(14.3)$ & $1.667(0.261-10.638)$ & 0.589 \\
\hline Posttraumatic arthritis & $1(0.8)$ & $0(0)$ & 0 & 1.000 \\
\hline Sequelae of hip pyogenic arthritis & $1(0.8)$ & $0(0)$ & 0 & 1.000 \\
\hline Displaced femoral neck fracture & $4(3.6)$ & $1(7.1)$ & $0.833(0.082-8.433)$ & 0.877 \\
\hline Operative femoral anteversion (degree) & $13.06 \pm 11.11$ & $23.43 \pm 13.50$ & $1.069(1.021-1.118)$ & 0.004 \\
\hline Distribution of anteversion, n (\%) & & & & $<0.001$ \\
\hline$<10^{\circ}$ & $40(35.7)$ & $2(14.3)$ & Reference & \\
\hline $10-30^{\circ}$ & $67(59.8)$ & $7(50)$ & $2.090(0.414-10.554)$ & 0.372 \\
\hline$>30^{\circ}$ & $5(4.5)$ & $5(35.7)$ & $20.000(3.036-131.731)$ & 0.002 \\
\hline
\end{tabular}

$\mathrm{Cl}$ confidence interval, $\mathrm{OR}$ odds ratio, $\mathrm{BMI}$ body mass index, $\mathrm{DDH}$ developmental dysplasia of the hip

measured operative femoral anteversion not for femur first technique, but aimed to identify the abnormal native anteversion. We did not recommend adjusting the cup anteversion in tune with varying femoral anteversions. In cases with large native femoral anteversion, decreasing the cup anteversion can cause anterior protrusion of the cup due to the achievement of optimally combined anteversion. And impingement between the iliopsoas tendon and the anterior edge of the cup is a potential cause of groin pain and functional limitations after THA [29]. On the femoral side, we can adjust the stem anteversion to a physiologically normal value according to operative femoral anteversion. So we thought the operative anteversion was more meaningful for the planning of THA. To predict the position of the femoral stem, Park et al. built the relationships between native femoral anteversion on different CT sections and postoperative stem anteversion [13]. However, the version on the cutting surface was more visualized than the measurement results from preoperative CT scans. Influenced by the various lateral inclination of the femur, we can hardly make the measurement on a consistent CT section. Besides, the midcortical line of the cutting surface varied with the cutting height, which also increased the difficulty for preoperative estimation on CT scan $[14,30]$. Therefore, we supposed that intraoperative estimation of femoral anteversion cannot be totally replaced by preoperative measurement. Several studies have found the lesser trochanter a reliable bony landmark. Based on a CT scan, Unlu et al. found a constant relationship between the version of less trochanter and posterior femoral condyles [31]. Shon et al. also observed a stable intersection angle between the posterior lesser trochanter line and femoral neck axis [32]. However, Worlicek et al. found significant differences in gender and left/right side when evaluating the correlation between the posterior lesser trochanter line and the posterior femoral condyle axis [33]. They concluded that posterior lesser trochanter line should not be used to determine femoral anteversion in CT scan. Moreover, different from measurement in the CT section, the contour of the lesser trochanter was difficult to determine due to its irregular morphology during THA, which further reduced its utility.

The operative femoral anteversion we measured ranged from -9 to $60^{\circ}$, with a large variation. There were not many results from previous literatures to compare. Researchers paid more attention to the estimation of stem anteversion, not the version of cutting surface $[7$, $15,16,18-20,34]$. Based on their results of stem anteversion, we can also find a wide range of values, which deviate from the generally advised $10-20^{\circ}[16,18,19$, 34]. It reminded us that variations of femoral proximal anatomy could be encountered in patients requiring THA. Different from other studies, we further analyzed the characteristics of hips with different ranges of 
femoral anteversion. Significant differences were observed in the age and diagnosis between hips with femoral anteversion $>30^{\circ}$ and the other two groups. Femoral anteversion $>30^{\circ}$ was more common in patients with a younger age and the diagnosis of DDH. This can be explained by the clinical features of DDH, including younger age when receiving THA and generally excessive femoral anteversion, especially for hips of low to high dislocation [35]. With regard to osteonecrosis, femoral anteversion $<10^{\circ}$ was seen in $15 / 49$ hips, for whom care should be taken to prevent from inserting a relative retroverted stem. Generally, the anteversion of cementless femoral stem was thought to be dictated by the native proximal femoral anatomy, with less ability to adjust. But the tapered wedge stems we used in our cohort were demonstrated more flexible in rotation compared with metaphyseal fit stem $[25,26]$. When there was a need of $>10^{\circ}$ adjustment, we preferred to choose the neckadjustable stems. In this study, there were totally 14 hips treated with the neck-adjustable femoral stem during THA. Among them, there were 7 sleeve modular components and 7 monoblock stem with three different neck versions. We further analyzed the influencing factors for the need of neck-adjustable femoral stem. Based on the results of univariate analysis, we found that female sex, diagnosis of DDH (compared with osteonecrosis), and higher operative femoral anteversion and its value $>30^{\circ}$ (compared with $<10^{\circ}$ ) were correlated with higher rates of using the neck-adjustable femoral stem. In sum, the primary influencing factor was the excessively larger femoral anteversion, which was more common in patients of DDH [35]. The discrepancy in sex can also be explained by the higher frequency of DDH pathology in women [36]. However, all factors lost statistical significance in the multivariate model. We supposed that it might be associated with the small sample size in the group of using neck-adjustable stem, which made the multivariate model unpowered to identify the important influencing factors. Moreover, apart from the concern about larger femoral anteversion, we should also actively deal with the anteversion $<10^{\circ}$ or even retroversion to avoid anterior impingement.

\section{Conclusions}

This study introduced a newly developed goniometer to measure femoral anteversion intraoperatively and highlighted the significance of operative femoral anteversion. Identification of abnormal femoral anteversion could assist in adjusting stem anteversion and reduce the risk of dislocation after THA.

\section{Abbreviations}

THA: Total hip arthroplasty; DDH: Developmental dysplasia of the hip; BMI: Body mass index; OR: Odds ratio; Cl: Confidence interval
Acknowledgements

The authors would like to thank all staff from the participating departments and clinics.

\section{Authors' contributions}

JS analyzed the data and drafted the manuscript. BZ collected the data and drafted the manuscript. LG and QZ assessed the radiographs and collected the data. WC performed the statistical analysis and interpreted the data. JL followed up the patients, collected the data, and analyzed the data. MN created the concept, performed the surgery, and revised the manuscript. GZ participated in the design of the study, performed the surgery, and revised the manuscript. All authors read and approved the final manuscript.

\section{Funding}

This research did not receive any specific grant from funding agencies in the public, commercial, or not-for-profit sectors.

\section{Availability of data and materials}

All data generated or analyzed during this study are included in this published article.

\section{Declarations}

Ethics approval and consent to participate

This retrospective review study involving human participants was in accordance with the ethical standards of the institutional and national research committee and with the 1964 Helsinki Declaration and its later amendments or comparable ethical standards. The study was approved by the medical ethics committee of our hospital. Informed consent was obtained from all individual participants included in the study.

\section{Consent for publication}

Not applicable.

\section{Competing interests}

The authors declare that they have no competing interests.

\section{Author details}

'Medical School of Chinese PLA, Beijing 100853, China. ${ }^{2}$ Department of Orthopedics, the First Medical Center, Chinese People's Liberation Army General Hospital, Fuxing Road, Haidian District, Beijing 100853, China.

Received: 15 March 2021 Accepted: 23 May 2021

Published online: 31 May 2021

\section{References}

1. Miki H, Sugano N. Modular neck for prevention of prosthetic impingement in cases with excessively anteverted femur. Clin Biomech (Bristol, Avon). 2011;26:944-9.

2. Ezquerra $L$, Quilez $M$, Pérez $M$, et al. Range of movement for impingement and dislocation avoidance in total hip replacement predicted by finite element model. J Med Biol Eng. 2017;37(1):26-34. https://doi.org/10.1007/s4 0846-016-0210-4

3. Elkins J, Callaghan J, Brown T. The 2014 Frank Stinchfield Award: the 'landing zone' for wear and stability in total hip arthroplasty is smaller than we thought: a computational analysis. Clin Orthop Relat Res. 2015;473(2): 441-52. https://doi.org/10.1007/s11999-014-3818-0.

4. Tezuka T, Heckmann N, Bodner R, et al. Functional safe zone is superior to the Lewinnek safe zone for total hip arthroplasty: why the Lewinnek safe zone is not always predictive of stability. J Arthroplast. 2019;34(1):3-8. https://doi.org/10.1016/j.arth.2018.10.034.

5. Abdel $M$, von Roth $P$, Jennings $M$, et al. What safe zone? The vast majority of dislocated THAs are within the Lewinnek safe zone for acetabular component position. Clin Orthop Relat Res. 2016;474(2):386-91. https://doi. org/10.1007/s11999-015-4432-5.

6. Matsushita A, Nakashima Y, Fujii M, Sato T, Iwamoto Y. Modular necks improve the range of hip motion in cases with excessively anteverted or retroverted femurs in THA. Clin Orthop Relat Res. 2010:468(12):3342-7. https://doi.org/10.1007/s11999-010-1385-6. 
7. Dorr L, Malik A, Dastane M, et al. Combined anteversion technique for total hip arthroplasty. Clin Orthop Relat Res. 2009;467(1):119-27. https://doi.org/1 0.1007/s11999-008-0598-4.

8. Widmer K. The impingement-free, prosthesis-specific, and anatomy-adjusted combined target zone for component positioning in THA depends on design and implantation parameters of both components. Clin Orthop Relat Res. 2020;478(8):1904-18. https://doi.org/10.1097/CORR.0000000000001233.

9. Uding A, Bloom N, Commean P, et al. Clinical tests to determine femoral version category in people with chronic hip joint pain and asymptomatic controls. Musculoskelet Sci Pract. 2019;39:115-22. https://doi.org/10.1016/j. msksp.2018.12.003.

10. Boissonneault A, Hiranaka T, Roberson J, et al. A validated single-view radiographic alternative to computed tomography for the measurement of femoral anteversion: a method-comparison study. J Arthroplast. 2017;32(3): 1018-23. https://doi.org/10.1016/j.arth.2016.09.016.

11. Liodakis E, Doxastaki I, Chu K, Krettek C, Gaulke R, Citak M, et al. Reliability of the assessment of lower limb torsion using computed tomography: analysis of five different techniques. Skelet Radiol. 2012;41(3):305-11. https://doi. org/10.1007/s00256-011-1185-4

12. Mao C, Liang Y, Ding C, Guo L, Wang Y, Zeng Q, et al. The consistency between measurements of the femoral neck anteversion angle in $\mathrm{DDH}$ on three-dimensional CT and MRI. Acta Radiol. 2016;57(6):716-20. https://doi. org/10.1177/0284185115603244.

13. Park K, Tsai T, Dimitriou D, et al. Utility of preoperative femoral neck geometry in predicting femoral stem anteversion. J Arthroplast. 2015;30(6): 1079-84. https://doi.org/10.1016/j.arth.2015.01.016.

14. Sariali $E$, Knaffo $Y$. Three-dimensional analysis of the proximal anterior femoral flare and torsion. Anatomic bases for metaphyseally fixed short stems design. Int Orthop. 2017:41(10):2017-23. https://doi.org/10.1007/s002 64-017-3435-0.

15. Fujihara Y, Fukunishi S, Fukui T, Nishio S, Okahisa S, Takeda Y, et al. Use of the G-guide for measuring stem antetorsion during total hip arthroplasty. Orthopedics. 2016;39(2):e271-5. https://doi.org/10.3928/01477447-2016020103.

16. Lee $Y$, Kim J, Kim T, et al. Validity of the intra-operative measurement of stem anteversion and factors for the erroneous estimation in cementless total hip arthroplasty using postero-lateral approach. Orthop Traumatol Surg Res. 2018;104(3):341-6. https://doi.org/10.1016/j.otsr.2017.11.023.

17. Murray D. The definition and measurement of acetabular orientation. J Bone Joint Surg Br. 1993;75:228-32.

18. Dorr L, Wan Z, Malik A, et al. A comparison of surgeon estimation and computed tomographic measurement of femoral component anteversion in cementless total hip arthroplasty. J Bone Joint Surg Am. 2009;91(11): 2598-604. https://doi.org/10.2106/JBJS.H.01225.

19. Wines A, McNicol D. Computed tomography measurement of the accuracy of component version in total hip arthroplasty. J Arthroplast. 2006;21(5): 696-701. https://doi.org/10.1016/j.arth.2005.11.008.

20. Pongkunakorn A, Palawong P, Chatmaitri S, Phetpangnga N. Use of a digital protractor and a spirit level to determine the intraoperative anteversion of femoral component during cemented hip hemiarthroplasty: a prospective clinical trial. Arch Bone Jt Surg. 2019;7(4):314-20.

21. Nam K, Tsai T, Dimitriou D, et al. Ipsilateral varus knee alignment correlates with increased femoral stem anteversion in primary total hip arthroplasty. Hip Int. 2016;26(2):175-9. https://doi.org/10.5301/hipint.5000325.

22. Fukunishi S, Nishio S, Fujihara Y, Okahisa S, Takeda Y, Fukui T, et al. Accuracy of combined anteversion in image-free navigated total hip arthroplasty: stem-first or cup-first technique? Int Orthop. 2016;40(1):9-13. https://doi. org/10.1007/s00264-015-2784-9.

23. Myers C, Laz P, Shelburne $K$, et al. The impact of hip implant alignment on muscle and joint loading during dynamic activities. Clin Biomech (Bristol, Avon). 2018;53:93-100

24. Uemura K, Atkins $\mathrm{P}$, Fiorentino $\mathrm{N}$, et al. Hip rotation during standing and dynamic activities and the compensatory effect of femoral anteversion: an in-vivo analysis of asymptomatic young adults using three-dimensional computed tomography models and dual fluoroscopy. Gait Posture. 2018;61: 276-81. https://doi.org/10.1016/j.gaitpost.2018.01.016.

25. Worlicek M, Weber M, Craiovan B, Wörner M, Völlner F, Springorum HR, et al. Native femoral anteversion should not be used as reference in cementless total hip arthroplasty with a straight, tapered stem: a retrospective clinical study. BMC Musculoskelet Disord. 2016;17(1):399. https://doi.org/10.1186/s12891-016-1255-9.
26. Marcovigi A, Ciampalini L, Perazzini P, Caldora P, Grandi G, Catani F. Evaluation of native femoral neck version and final stem version variability in patients with osteoarthritis undergoing robotically implanted total hip arthroplasty. J Arthroplast. 2019;34(1):108-15. https://doi.org/10.1016/j.arth.2 018.06.027.

27. Weber M, Woerner M, Craiovan B, Voellner F, Worlicek M, Springorum HR, et al. Current standard rules of combined anteversion prevent prosthetic impingement but ignore osseous contact in total hip arthroplasty. Int Orthop. 2016;40(12):2495-504. https://doi.org/10.1007/s00264-016-3171-X.

28. Ohmori T, Kabata T, Kajino Y, Inoue D, Taga T, Yamamoto T, et al. The optimal combined anteversion pattern to achieve a favorable impingement-free angle in total hip arthroplasty. J Orthop Sci. 2019;24(3): 474-81. https://doi.org/10.1016/j.jos.2018.11.008.

29. Park KK, Tsai TY, Dimitriou D, Kwon YM. Three-dimensional in vivo difference between native acetabular version and acetabular component version influences iliopsoas impingement after total hip arthroplasty. Int Orthop. 2016;40(9):1807-12. https://doi.org/10.1007/s00264-015-3055-5.

30. Tsukeoka T, Tsuneizumi Y, Lee T. The T-line as an intraoperative landmark for reproducing the native femoral anteversion during hip arthroplasty. Arch Orthop Trauma Surg. 2014;134(6):873-9. https://doi.org/10.1007/s00402014-1978-8

31. Unlu M, Kesmezacar $\mathrm{H}$, Kantarci $\mathrm{F}$, et al. Intraoperative estimation of femoral anteversion in cementless total hip arthroplasty using the lesser trochanter. Arch Orthop Trauma Surg. 2011;131(9):1317-23. https://doi.org/10.1007/s004 02-011-1282-9.

32. Shon W, Yun H, Yang J, et al. The use of the posterior lesser trochanter line to estimate femoral neck version: an analysis of computed tomography measurements. J Arthroplast. 2013;28(2):352-8. https://doi.org/10.1016/j.a rth.2012.03.001.

33. Worlicek M, Weber M, Craiovan B, Zeman F, Grifka J, Renkawitz T, et al. Posterior lesser trochanter line should not be used as reference for assessing femoral version in CT scans: a retrospective reliability and agreement study. Acta Radiol. 2017;58(9):1101-7. https://doi.org/10.1177/02 84185116682383.

34. Hirata M, Nakashima Y, Ohishi M, Hamai S, Hara D, Iwamoto Y. Surgeon error in performing intraoperative estimation of stem anteversion in cementless total hip arthroplasty. J Arthroplast. 2013;28(9):1648-53. https:// doi.org/10.1016/j.arth.2013.03.006

35. Greber E, Pelt C, Gililland J, et al. Challenges in total hip arthroplasty in the setting of developmental dysplasia of the hip. J Arthroplast. 2017;32(9):S3844. https://doi.org/10.1016/j.arth.2017.02.024.

36. Kosuge D, Yamada N, Azegami S, Achan P, Ramachandran M. Management of developmental dysplasia of the hip in young adults: current concepts. Bone Joint J. 2013;95-B(6):732-7. https://doi.org/10.1302/0301-620X.95B6.312 86.

\section{Publisher's Note}

Springer Nature remains neutral with regard to jurisdictional claims in published maps and institutional affiliations.

\section{Ready to submit your research? Choose BMC and benefit from:}

- fast, convenient online submission

- thorough peer review by experienced researchers in your field

- rapid publication on acceptance

- support for research data, including large and complex data types

- gold Open Access which fosters wider collaboration and increased citations

- maximum visibility for your research: over $100 \mathrm{M}$ website views per year

At $\mathrm{BMC}$, research is always in progress.

Learn more biomedcentral.com/submission 